\title{
Non traumatic lesions of the scapula-Pictorial essay
}

\author{
Subbarao Kakarla ${ }^{1, *}$
}

${ }^{1}$ Chairman, KIMS Foundation and Research Centre, Minister Road, Secunderabad - 500003, Telangana, India

\begin{abstract}
The scapula is a flat, broad and triangular bone situated bilaterally over the chest wall. The division of the scapula into two anatomical areas helps the imageologist in deciding the nature of tumors and tumor like lesions. It also helps the surgeon in choosing the type of surgery. Non traumatic lesions of the scapula include developmental/ congenital, inflammatory, infective, metabolic, neoplastic and miscellaneous causes. The images of tumor like lesions such as infections, Langerhans histiocytosis, simple bone cysts, congenital glenoid dysplasia, fibrous dysplasia and Paget disease are described. Few of the metabolic and systemic disorders are also outlined with illustrations. Similarly, the common benign and malignant tumors as well as their characteristics are described and illustrated. The imaging features of tumors are more helpful in the diagnosis by analysing the site, transitional zone, aggressive nature, matrix mineralisation, periosteal reaction and soft tissue changes.
\end{abstract}

Keywords: Imaging of scapula; inflammatory; neoplastic lesions; scapula; tumors of scapula

*Corresponding author: Prof. Kakarla Subbarao, MS, D.Sc. (HON), FRCR, FACR, FICP, FSASMA, FCCP, FICR, FCGP, Chairman, KIMS Foundation and Research Centre, Minister Road, Secunderabad 500003, Telangana, India. Email: subbaraokakarla25@gmail.com

Received 27 December 2018; Accepted 15 March 2019; Published 22 March 2019

Citation: Kakarla S. Non traumatic lesions of the scapula-Pictorial essay. J Med Sci Res. 2019; 7(2):51-60. DOI: http://dx.doi. org/10.17727/JMSR.2019/7-10

Copyright: (c) 2019 Kakarla S. Published by KIMS Foundation and Research Center. This is an open-access article distributed under the terms of the Creative Commons Attribution License, which permits unrestricted use, distribution, and reproduction in any medium, provided the original author and source are credited.

\section{Introduction}

The scapula (shoulder blade) is a flat, broad and triangular bone, which lies obliquely over the posterior aspect of the chest wall extending over the first seven ribs bilaterally. It consists of two surfaces, three borders and three angles. Enneking et al. [1], have given a classification system for diagnosing the tumors of the shoulder girdle. Accordingly, the scapula is divided into two zones, one, the acromio glenoid complex designated as S2 region and two, the spine and blade portion as S1 region, as illustrated in (Figure 1). This system helps the surgeon for resections and reconstructions.

The tumors of the $\mathrm{S} 1$ region are those that commonly develop in flat bones such as, osteosarcoma, round cell tumor, chondrosarcoma and multiple myeloma. The tumors that develop in S2 region include those that commonly develop at the ends of the long bones, which include osteochondroma, giant cell 
tumor and chondroblastoma. Other focal lesions include developmental, infective, Langerhans cell histiocytosis and other benign miscellaneous lesions such as, hydatid cyst, radiation induced malignancies etc. Systemic benign disorders, such as skeletal dysplasias, metabolic and haematological may also involve the scapula (Table 1).

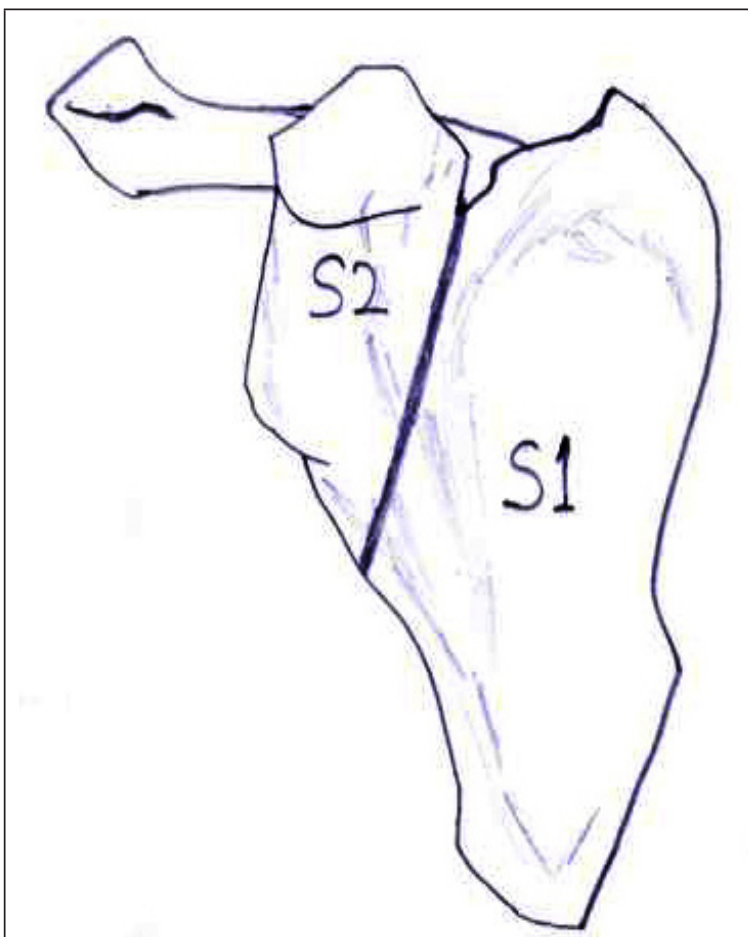

Figure 1: Diagram showing the system for classification of skeletal resection advised by committee of Muskuloskeletal Tumor Society. S1 - Blade of scapula, S2 - Glenoid acromial complex.

Table 1: Lesions of the scapula: Focal and systemic.

\begin{tabular}{|llll|}
\hline Focal & \multicolumn{2}{l|}{ Systemic } \\
\hline - & Developmental & - & Dysplasias \\
- & Inflammations & - & Metabolic \\
- & Infections & - & Hematological \\
- & Neoplastic - benign & - & Neoplastic - Malignant, \\
& \& malignant & & metastasis, myeloma \\
& & and lymphoma \\
\hline
\end{tabular}

The simple and most preferred investigation is conventional radiography. However, recent imaging modalities such as, MDCT, MRI, scintigraphy and PET$\mathrm{CT}$ are adopted to arrive at a definitive diagnosis. The imaging methods are listed in Table 2. The benign neoplasms are listed in Table 3, tumor like lesions in Table 4 and malignant lesions in Table 5.
Table 2: Imaging methods.

$$
\begin{array}{ll}
\text { - } & \text { Conventional/Digital } \\
\text { - } & \text { MDCT } \\
\text { - } & \text { MRI } \\
\text { - } & \text { Radionuclide Scan } \\
\hline
\end{array}
$$

\section{Review of the literature}

The literature is scanty regarding the tumors and tumoral lesions of scapula. However Enneking et al. [1] mentioned the division of scapula into two major areas which helps the surgeon for resections and reconstructions of the tumors. Blacksin et al. [2] described the radiological findings of neoplasms of the scapula. Tumoral conditions were included in the article by Brtkova et al. [3]. Several other authors have mentioned individual entities and described the radiological findings but not in a consolidated fashion.

\section{Discussion}

Tumors of the scapula are relatively rare. Statistically, most frequently encountered include are tumours of cartilaginous origin. In our experience, more than $1 / 3$ of all cases are osteochondromas, which show a typical appearance radiologically. These are encountered predominantly in the first 3 decades in males and are located most often in the body of the scapula. The other benign tumors include osteoma, osteoid osteoma, osteoblastoma, hemangioma, aneurysmal bone cyst and giant cell tumor (Table 3 ). The tumoral conditions include cystic type of osteomyelitis, Langerhans cell histiocytosis and simple bone cyst. Radiological manifestations have been described by several authors [4-7]. The most common malignant lesion of the scapula is chondrosarcoma, which generally occurs in the 4th-7th decades of age. Chondrosarcoma is located mainly at the lateral scapular margin over the inferior angle, in the acromion and coracoid process and their appearances range from typical characteristics to falsely benign lesions. The other primary malignant tumors include osteosarcoma, Ewing's sarcoma, plasmacytoma, lymphoma, hemangioendothyleoma and malignant fibrous histiocytoma. Of all the malignant bone tumors, metastases are the most common. The primary sites include majorly, carcinoma of the breast, lung, kidney and thyroid [8]. Infact, any primary malignancy from any organ can produce metastases in the 
bones. The radiological appearances vary from lytic, mixed to diffusely sclerotic. Sclerotic metastases are primarily from carcinoma of the prostate, but can be from carcinoids, malignancies from gastrointestinal and genitourinary tracts. Soft tissue lesions such as, fibromatosis may involve scapula [9]. Metabolic and endocrine disorders may reflect upon scapula [10]. Hemoglobinopathies and malignancies of the hemopoietic system, eg. Leukemia and lymphoma may involve the scapula. Miscellaneous lesions include Paget disease. These may be confined to scapula but most often these are polyostotic [11, 12].

\section{Imaging spectrum:}

Table 3: Benign bone tumors. large nidus more than $2 \mathrm{cms}$ in diameter. The nidus may be obscured by new bone. It is rare in the scapula. CT is essential to confirm the diagnosis (Figure 3). There is no preferential site for this in the scapula.

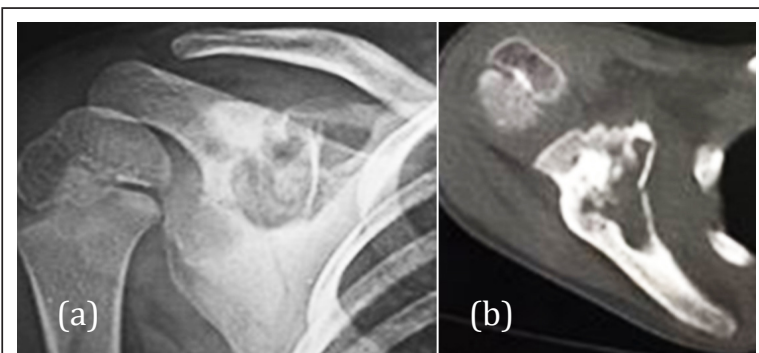

Figure 3: Osteoblastoma, (a) Plain radiograph, (b) CT showing the large nidus and new bone formation.

\section{Osteochondroma}

This is the most common primary benign neoplasm of the scapula. Usually, it is a single lesion located on the anterior surface of the scapular body like an osteocartilaginous exostosis. The radiologic presentation of osteochondroma is characterized depending on whether the lesion is pedunculated with a narrow pedicle or, sessile with a broad base of implantation in the cortex. The most important feature is the long continuity of the cortical bone with the cortex of osteochondroma (Figure 4a-c). Other important features are calcifications in the cartilaginous cap with a thickness which ranges from $1 \mathrm{~mm}-3 \mathrm{~mm}$. This is better displayed on MRI as a cap of high intensity in gradient echo sequences and $\mathrm{T} 2$ weighted images. Osteochondroma may be a part of hereditary multiple exostoses (Figure 4 d, e). Complications of osteochondroma include compression of nerves, blood vessels, fracture and bursal formation (Figure 4f). Deformity and malignant transformation to chondrosarcoma are the other complications. Enchondromas and other cartilaginous lesions such as, chondroblastoma rarely involve the scapula.

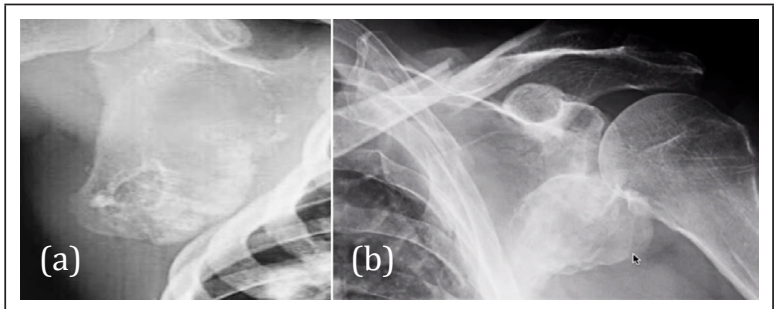

Figure 4a, b: Sessile osteochondroma of the scapula.

\section{Osteoblastoma}

It produces excessive new bone formation with a 


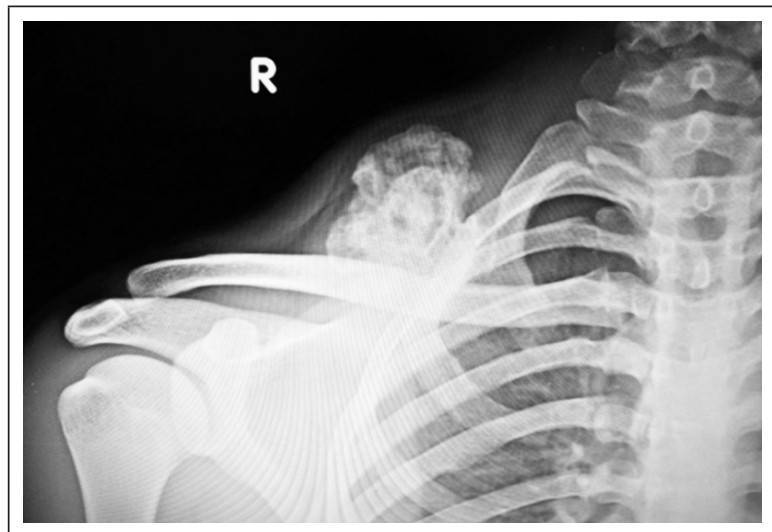

Figure 4c: Osteochondroma arising from the scapula.

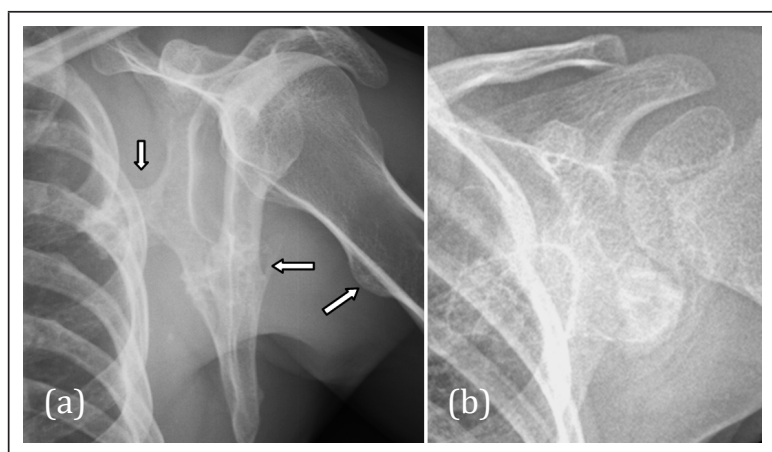

Figure 4d, e: Multiple hereditary exostoses involving the scapula and humerus.

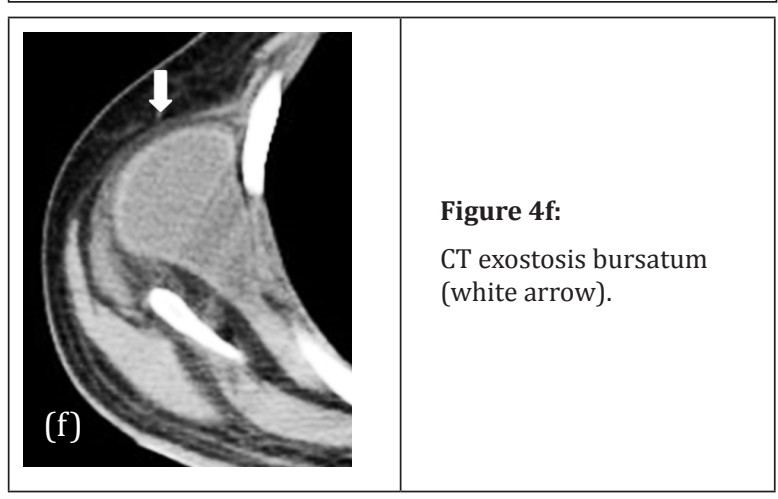

\section{Hemangioma}

It is a benign vascular tumor, which commonly occur in the vertebral bodies and skull. They may be capillary, cavernous, or venous. In the skull, they produce a spoke wheel appearance. Hemangiomas of the scapula are rare and the capillary hemangiomas produce the same radiological appearance as seen elsewhere. Cavernous hemangiomas produce large lucent areas with little surrounding sclerosis (Figure 5a). Scapula may be involved in cystic angiomatosis of the skeleton which is a diffuse systemic disorder. Multiple lucent areas are noted with surrounding sclerosis (Figure $5 b$ ).

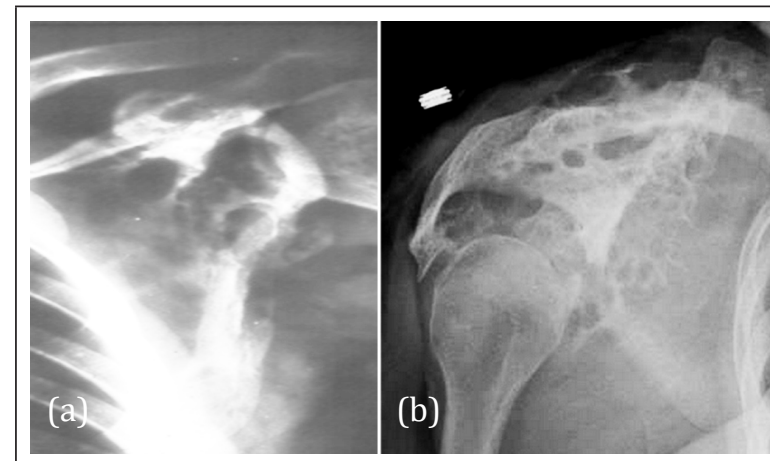

Figure 5a, b: (a) Cavernous hemangioma, (b) Cystic angiomatosis.

\section{Aneurysmal bone cyst (ABC)}

This accounts for $6 \%$ of primary lesions of bone. It occurs in children younger than 20 years old. Histologically, it consists of multiple sinusoids filled with blood, alternating with more solid areas. Multicystic eccentric lucencies involves the bone with a thin shell of periosteal response. Conventional radiography may be sufficient for diagnosis (Figure $6 a, b)$. But the use of MDCT allows us to assess the cortical bone and fine internal septa (Figure 6c-f). MRI findings are quite characteristic showing a well-defined lesion with a lobulated edge and cystic cavities with fluid-fluid levels (Figure 6g-i). Secondary ABC's may be encountered in giant cell tumors, chondroblastoma and some malignant tumors, such as telangiectatic osteosarcoma.

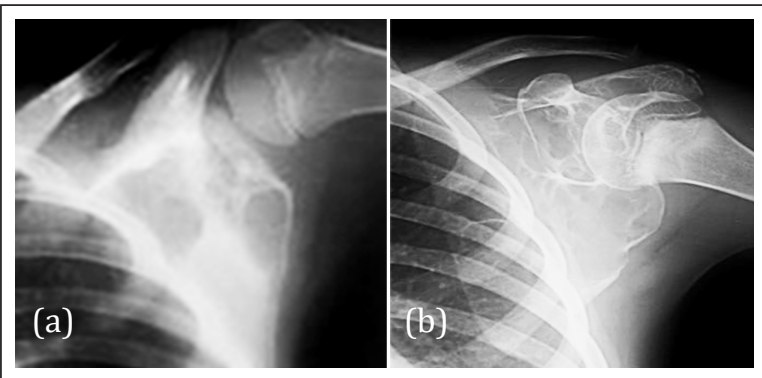

Figure $6 \mathbf{a}$, b: $\mathrm{ABC}$ of scapula.

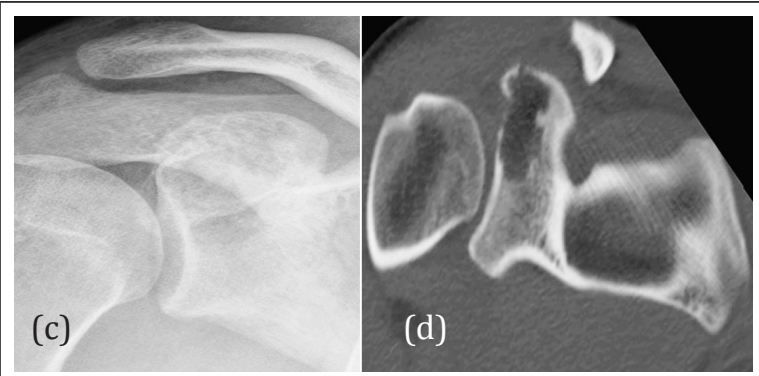

Figure 6c, d: ABC of the glenoid; (c) Plain, (d) CT. 


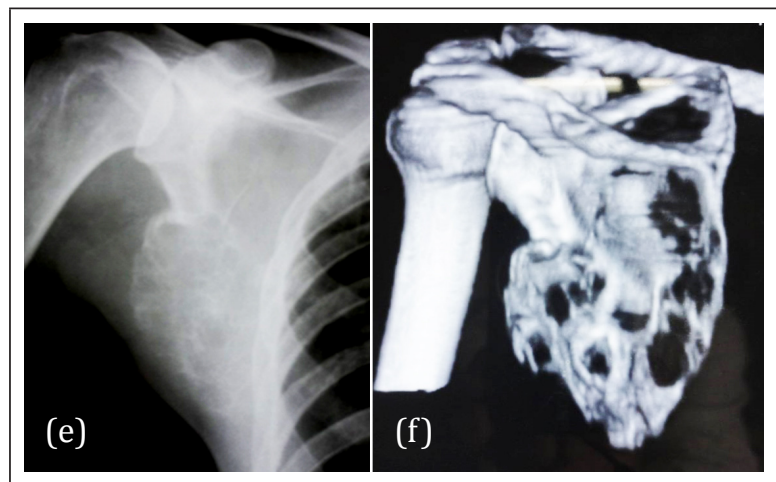

Figure 6e, f: ABC; (e) Plain film, (f) 3d CT.

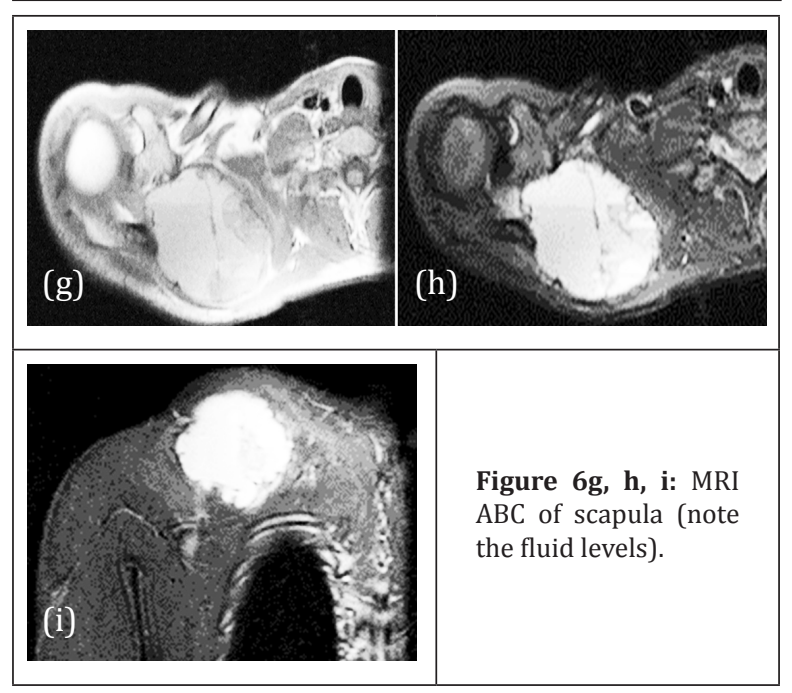

\section{Giant cell tumor}

It is an aggressive lesion characterized by a highly vascularized tissue containing stromal cells and osteoclasts. It affects men and women between 20 40 years old, although more common in women. Radiologically displayed an osteolytic lesion with a thin margin and narrow-transition. In general no periosteal reaction is noted unless there is a fracture. There is usually a soft tissue mass when the cortex is broken. CT and MRI play an important role in studying the matrix and soft tissue components. These benign bone tumors generally occur in the ends of long bones. In the flat bones they occur near the articular margins. Radiologically a lucent lesion with trabeculae in the matrix giving a soap bubble appearance is noted. They may be aggressive with cortical disruption with a pathological fracture. The margins of the lesion are rarely sclerotic (Figure 7a, b). In the differential diagnosis, aneurysmal bone cyst and chondroblastoma are to be considered. Infact, giant cell tumor and aneurysmal bone cyst may coexist in about $20 \%$ of cases.

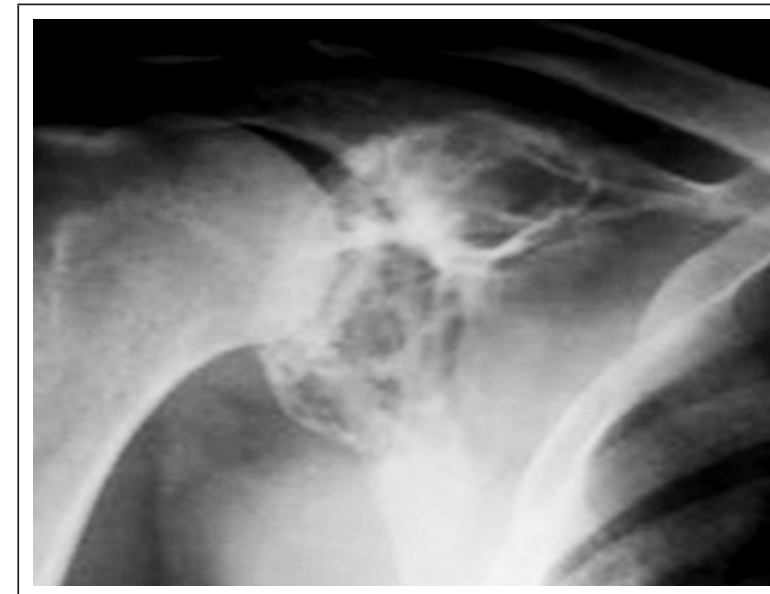

Figure 7a: 30M, Giant cell tumor involving the glenoid.

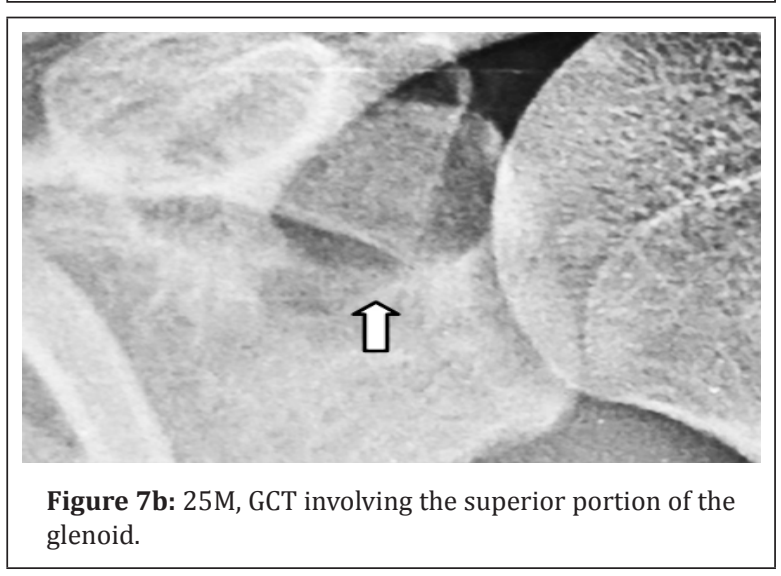

\section{Neurofibroma/ Schwannoma}

These are very rare tumors primarily arising from the scapula. However, in neurofibromatosis 1 (NF1) the scapula may be involved with associated mesenchymal dysplasia. Localised neurofibroma may produce irregular lucent defect in the scapula with variable surrounding sclerosis. It is a histological diagnosis. However, in NF1 patients, it can be diagnosed because of the associated findings in the rest of the body. Distortion of the architecture of the scapula is noted with variable lucencies with surrounding sclerosis (Figure 8a, b).

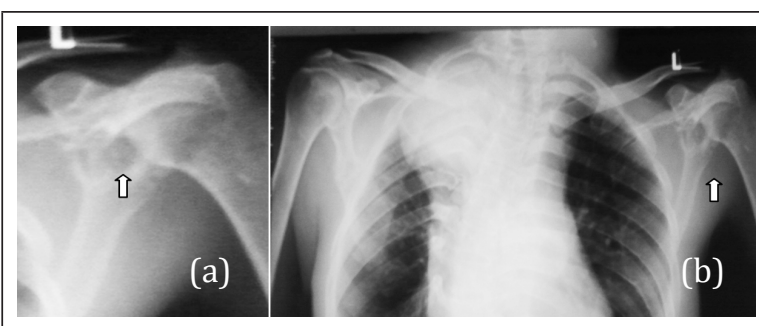

Figure 8a, b: (a) Neurofibroma of left scapula, (b) same patient chest in NF1. 
Table 4: Primary malignant neoplasms of the scapula.

- Osteosarcoma

- $\quad$ Ewing sarcoma

- Chondrosarcoma

- Fibrosarcoma/ Malignant fibrous histocytoma

- Lymphoma

- Plasmacytoma/ Multiple myeloma

- Hemangioendothelieoma

\section{Osteosarcoma}

It is a relatively frequent malignant neoplasm of the scapula. Occurs between 11 and 30 years. Several histological types, such as chondroblastic, osteoblastic and telangiectatic types have been reported. Radiological features include an amorphous, osteoid tumor and bone density with bulky soft-tissue masses (Figure 9a, b). Sun ray spicules may extent to the soft tissues. It may be lytic with broken cortex and extend into the soft tissues.
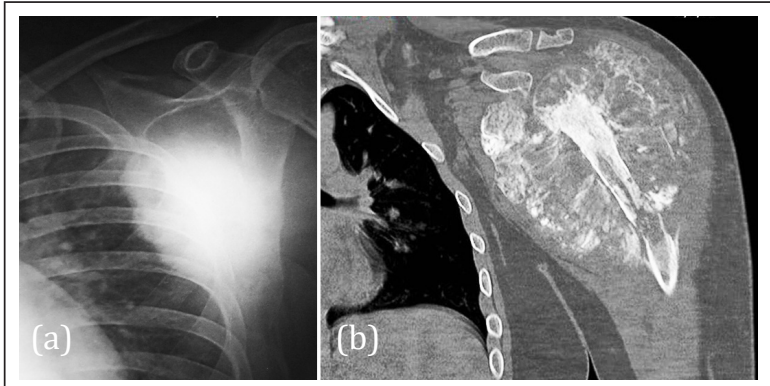

Figure 9a, b: Osteosarcoma of the scapula; (a) Osteoblastic, (b) Classical.

\section{Chondrosarcoma}

It is the most common malignant neoplasm of the scapula. Chondrosarcoma is a malignant bone tumor producing cartilage. Its main radiological features include an expansive lesion, disruption of bone, annular calcifications and comma-shaped tumor matrix, thickening of the bone cortex, and the presence of soft tissue mass (Figure 10a, b). Secondary chondrosarcoma arising from exostosis may be seen but radiological characteristics include the appearance of the pre existing lesion with a thick cartilage (Figure 10c).

\section{Ewing sarcoma}

This is commonly encountered in the long bones and pelvic bones. It may be encountered in the scapula. The age is between 5-30 years. From the radiological point of view it is permeable lytic lesion with a large soft tissue mass. Reactive new bone may be seen (Figure 11a, b). Soft tissue mass is better demonstrated by CT/MRI (Figure 11c).

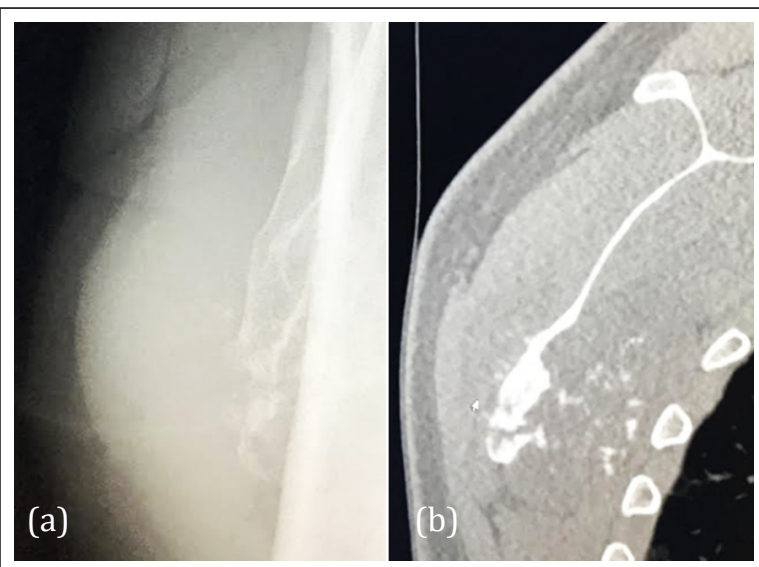

Figure 10a, b: Primary chondrosarcoma of the scapula; (a) Tangential plain film, (b) CT.
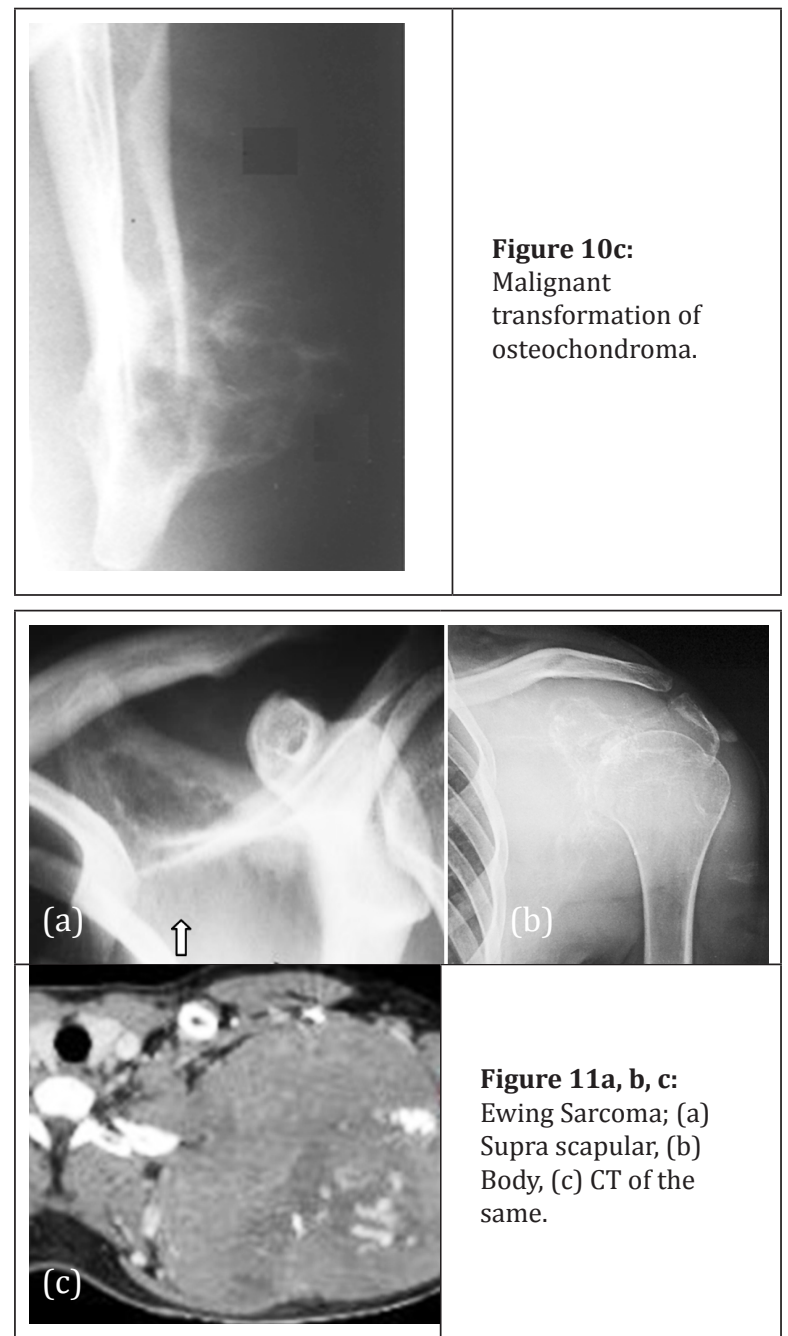


\section{Fibrosarcoma/ histiocytoma}

Malignant

fibrous

Most of the malignant fibrous histiocytomas were designated as fibrosarcomas in the previous years. It is a primary bone tumor of the appendicular skeleton and can occur at any age Radiological features are non specific which include a lytic lesion with cortical destruction and soft tissue mass. It is difficult to differentiate from fibrosarcoma (Figure 12). On MR imaging, malignant fibrous histiocytoma, often shows only heterogeneously increased signal intensity on $\mathrm{T} 2$-weighted sequences.

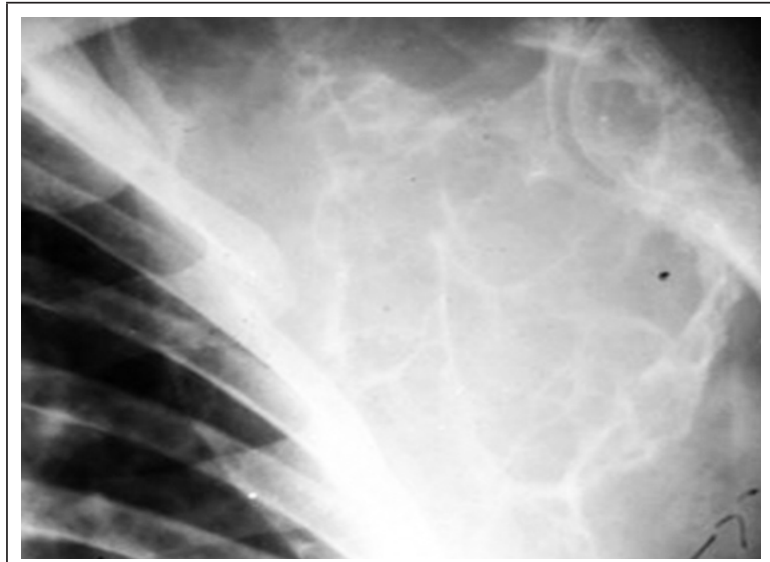

Figure 12: Malignant fibrous histiocytoma.

\section{Multiple myeloma}

This originates from plasma cells of the bone marrow. This is the most common primary malignant bone tumor. It occurs between 50-70 years of age. From the radiological point of view a localised form known as plasmacytoma shows a large lytic lesion with well-defined borders. Multiple myeloma is more commonly encountered than plasmacytoma in scapula. Multiple well defined lytic lesions, diffuse infiltrative pattern and moth eaten lytic areas are common radiological findings (Figure 13a, b).
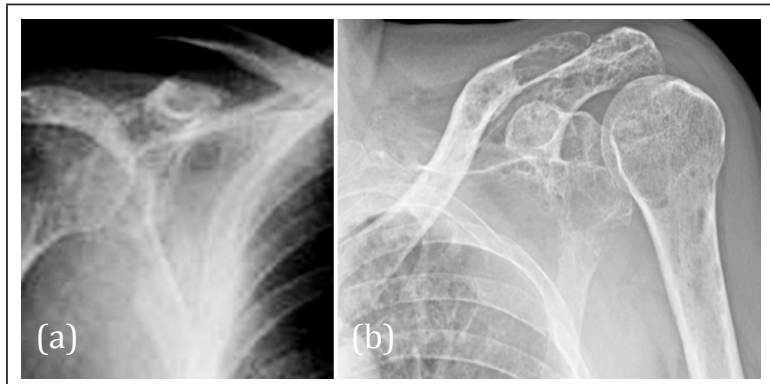

Figure 13a, b: Multiple myeloma involving the scapulae.

\section{Lymphoma, leukemia}

Isolated lymphoma/ leukemia involving the scapula is quite rare. However, scapular involvement in systemic lymphoma is occasionally noted. Based on radiological findings, it is difficult to make a firm diagnosis. It may be lytic, sclerotic or mixed (Figure $14 a, b, c)$.
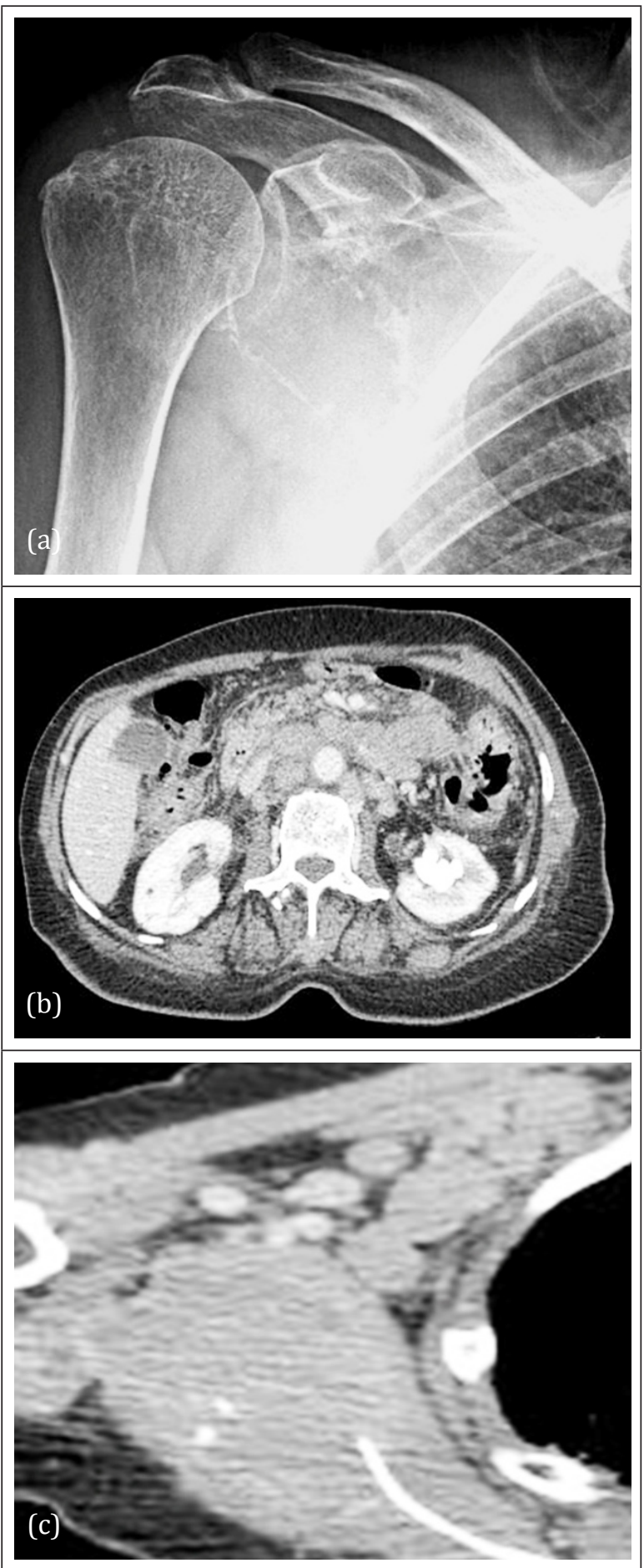

Figure 14a, b, c: Lymphoma; (a) Plain film, (b) CT showing para aortic enlarged nodes, (c) CT of scapula. 


\section{Metastatic disease}

They are the most common malignant bone tumors. It should always be taken into account in the differential diagnosis when dealing with malignant bone tumors, particularly in older patients. By frequency, carcinomas of the breast, lung, thyroid, kidney and prostate are responsible for most of the metastasis. While expanding osteolytic lesions are common from primary neoplasms of the kidney, lung, breast, thyroid and gastrointestinal tract, osteoblastic lesions are common in carcinoids, gastrointestinal tract and prostatic malignancies (Figure 15).

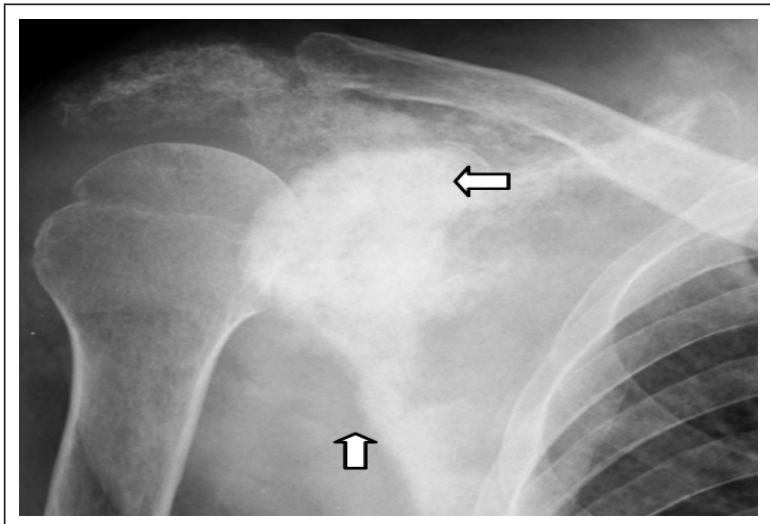

Figure 15: Prostatic metastasis to scapula.

Tumor like lesions of scapula are listed in Table 5.

Table 5: Tumor like lesions.

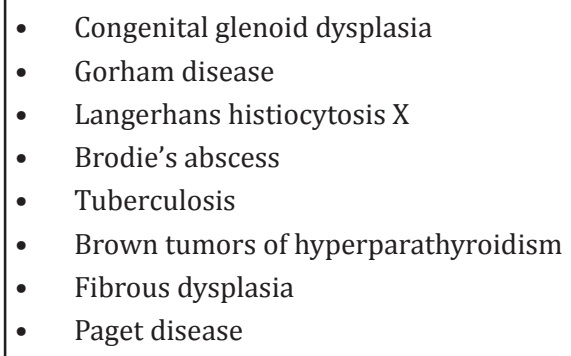

\section{Congenital glenoid dysplasia}

It can be unilateral or bilateral and the etiology is similar to acetabular dysplasia. It is characterized by underdevelopment of the bony glenoid and adjacent part of the scapular neck which may be seen as isolated. However, it is also noted in several syndromes and in patients with mucopolysaccharidoses. Radiologically, the findings vary with the degree of the dysplasia. The normal concavity of the glenoid fossa assumes a flat shape with a short neck, elongation of the glenoid and changes in the shape of the coracoid and clavicle. The glenoid typically shows notching and irregularity in its inferior aspect. In long standing cases secondary changes may occur in the head and neck of the humerus [9-11] (Figure 16a, b).

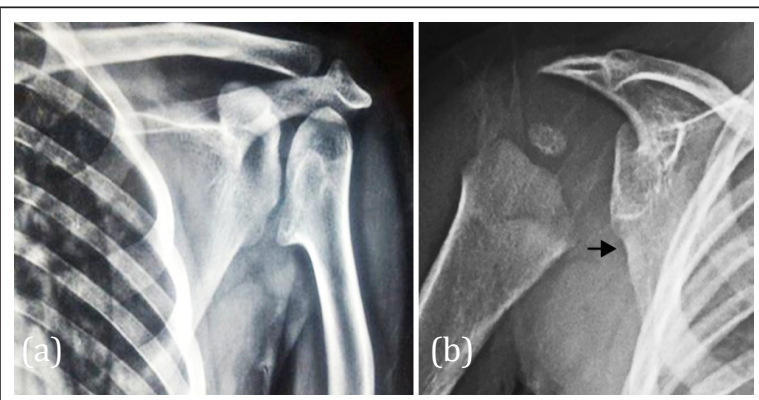

Figure 16a, b: Glenoid dysplasia: (b) 1-year-old - glenoid dysplasia (note the inferior notch).

\section{Gorham syndrome}

Gorham disease is also known as Gorham vanishing bone disease and phantom bone disease. It is a very rare skeletal lesion of unknown cause although vascular origin is postulated. Radiologically, extensive and progressive lytic lesions are noted which extend to the adjacent bones (Figure 17). These consist of thin-walled vascular or lymphatic channels within bone, which lead to resorption of bone.

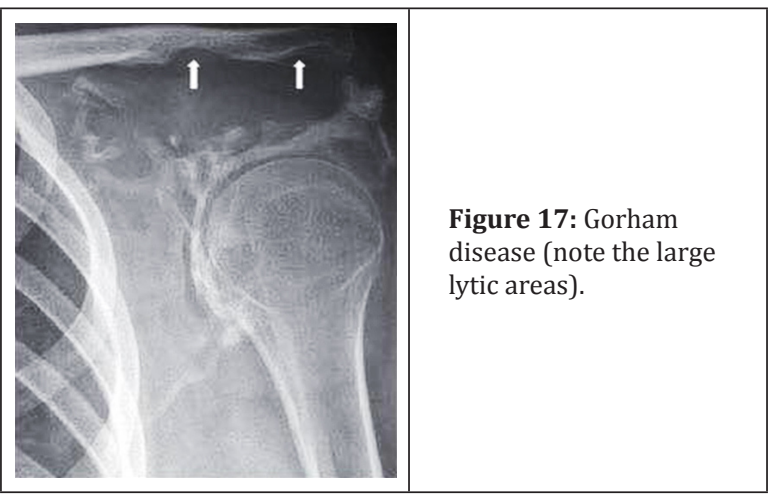

\section{Inflammatory}

Langerhans cells histiocytosis/ local manifestation as eosinophilic granuloma is a non-neoplastic proliferation characterized by granulomatous reticular cells. Usually occurs in children aged 1 to 15 years. The most common sites are the flat bones, spine, skull and long bones. These are lytic lesions, polyostotic, which may show an aggressive look like with destruction of cortical bone and soft 
tissue mass. CT demonstrates a lytic lesion with scalloped borders (Figure 18). MRI most often displays a focal lesion surrounded by a large area of alteration of signal due to a reaction of soft tissue and bone marrow, with low signal intensity on $\mathrm{T} 2$ is considered to correspond to bone marrow edema or parts soft due to the flare phenomenon.

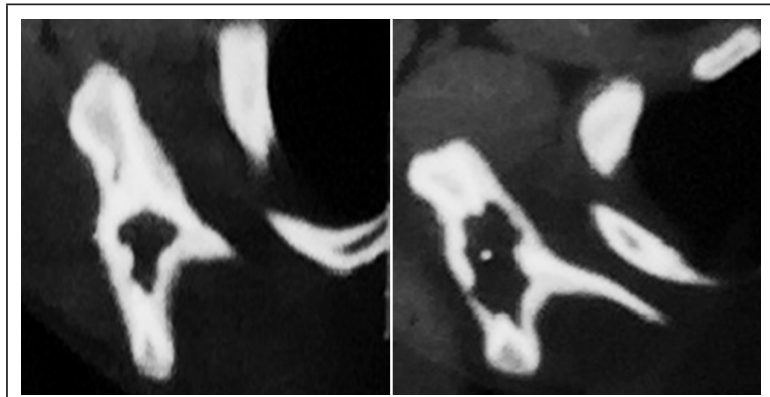

Figure 18: CT - Eosinophilic granuloma of scapula.

\section{Infections}

Infectious disease is represented by osteomyelitis of the scapula. It can be acute, subacute or chronic. Radiologically, the subacute form assumes Brodie's abscess with a central lucency surrounded by sclerosis (Figure 19a). The etiology may be bacterial or fungal (Figure 19b). Tuberculosis primarily involving the scapula is very rare. However, cystic type of tuberculosis may be encountered in children (Figure 19c).

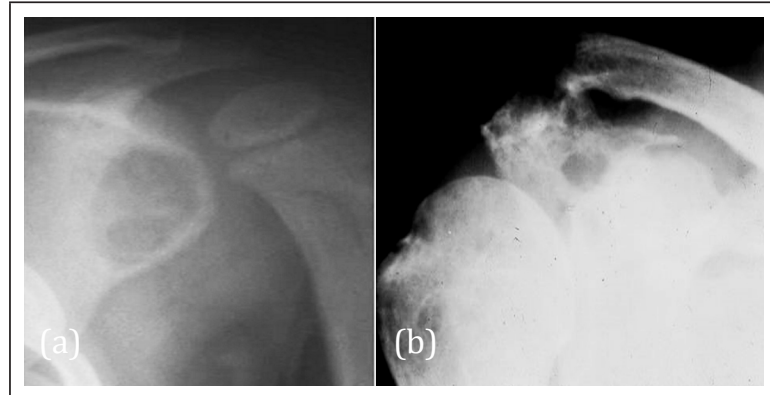

Figure 19a, b: (a) Subacute osteomyelitis of the scapula Brodie's abscess; (b) Actinomycosis.

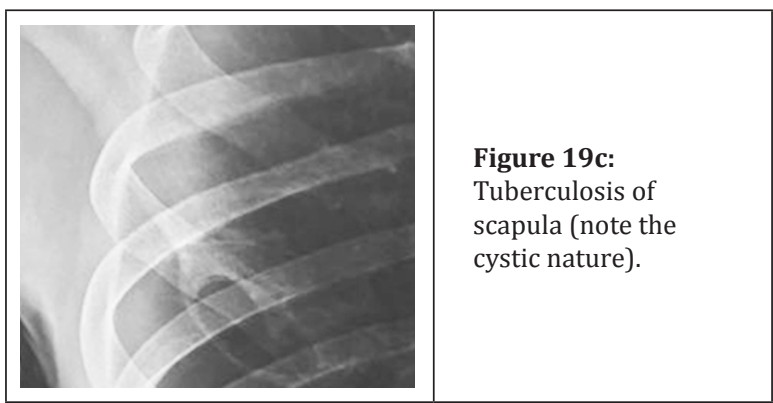

\section{Brown tumors}

In both primaryand secondary hyperparathyroidism, brown tumors in bones are encountered, although rare in scapula. Radiologically, a lytic lesion with ill-defined borders is noted in an osteoporotic bone (Figure 20a). In secondary hyperparathyroidism, brown tumors are associated with osteosclerosis (Figure 20b).

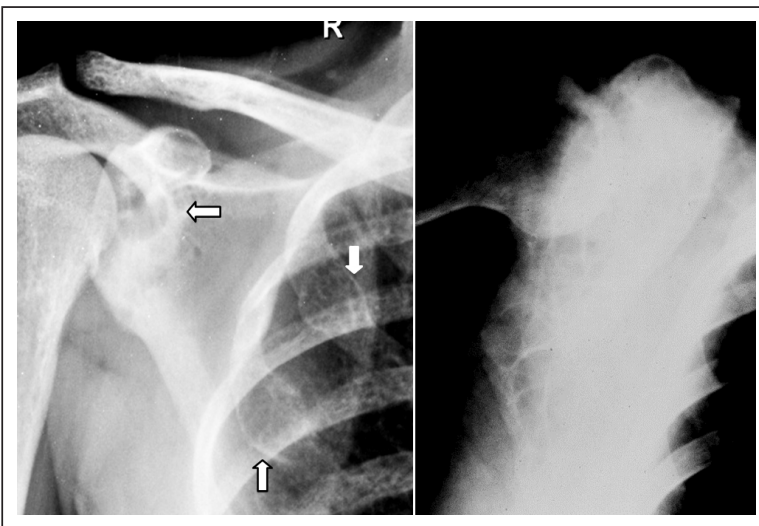

Figure 20: Brown Tumors of hyperparathyroidism; (a) Scapula and ribs, (b) Scapula.

\section{Fibrous dysplasia}

It is an aberrant formation of fibrous tissue in the bone and is developmental in origin. It can be monostotic, polyostotic or associated with other syndromes. Radiologically, it may appear as a lucent lesion with a sclerotic rind (Figure 21a, b). It may also be mixed, sclerotic and lucent (Figure 21c). Rarely, it is purely sclerotic with a matrix of groundglass appearance.

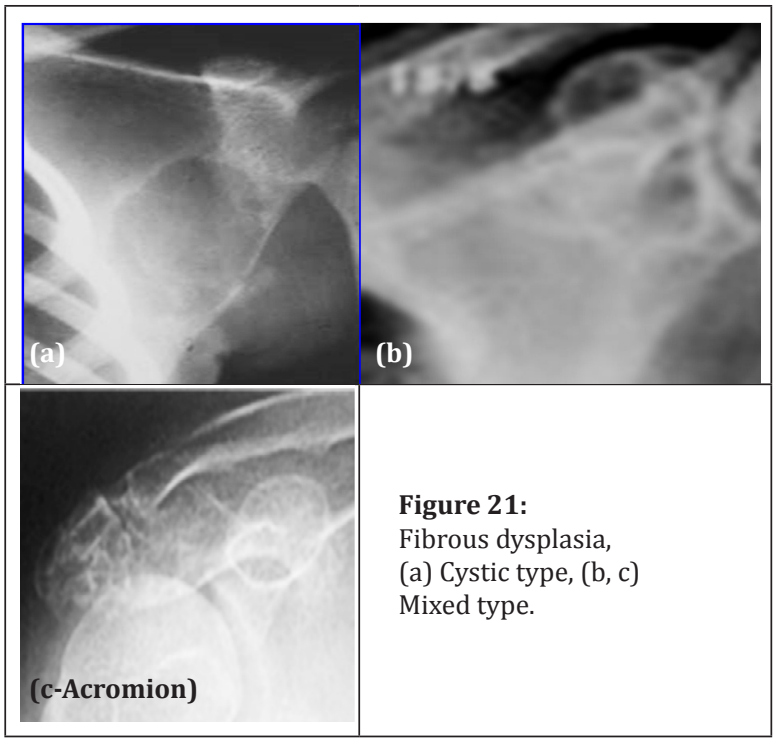




\section{Hemangiopericytoma}

This tumour is frequently encountered in middleaged adults $\left(4^{\text {th }}\right.$ decade). However, scapula is a rare site. Hemangiopericytoma, arises from the cells of Zimmerman, which surround vessels. Both benign and malignant forms are encountered. Radiologically the appearance varies from aneurysmal bone cyst to hemangioma of bone.

\section{Conclusion}

Non traumatic isolated scapular lesions are not uncommon. The etiology includes congenital, inflammatory, infective, neoplasms of benign and malignant nature. Systemic disorders include endocrinal, metabolic and neoplastic. Conventional radiography is adequate to make an initial diagnosis. However, advanced imaging combined with laboratory and histology findings is necessary to make a firm diagnosis. Imaging features of various entities involving the scapula are described with the help of illustrations.

\section{Acknowledgements}

NIMS, KIMS, KREST Museum Hyderabad, India.

\section{Conflicts of interest}

Author declares no conflicts of interest.

\section{References}

[1] Enneking W, Dunham W, Gebhardt M, Malawar M, Pritchard D. A system for the classification of skeletal resections. Chir Organi Mov 1990; 75(suppl 1): 217-240.

[2] Marcia MF, Joseph B. Neoplasms of the scapula - Pictorial essay; American Journal of Roentgenology, 2000; 174:17291735.

[3] Pourfeizi HH, Sales JG, Elmi A, Tabrizi A. Osteoid osteoma of a scapula: a case report in a 34 years old woman. Med J Islam Repub Iran. 2012; 26(3):143-146

[4] Sharma R, Weerakkody Y et al. Osteoblastoma; Radiopaedia, RID:10960.

[5] Brtková J1, Nidecker A, Zídková H, Jundt G. Tumours and tumour-like lesions of scapula, Acta Medica (Hradec Kralove). 1999; 42(3):103-110.

[6] Kakarla S. Benign Cartilaginous Lesions. J Med Sci Res. 2013; 1(1): 29-36.

[7] Nam SJ, Kim S, Lim BJ, Yoon CS, Kim TH, et al. Imaging of primary chest wall tumors with radiologic-Pathologic correlation. Radiographics. 2011; 31:749-770.

[8] Ogose A, Sim FH, O'Connor MI, Unni KK. Bone tumors of the coracoid process of the scapula. Clin Orthop Relat Res. 1999; 358:205-214

[9] Currarino G, Sheffield E, Twickler D. Congenital glenoid dysplasia. Pediatr Radiol. 1998; 28(1):30-37.
[10] Wirth MA, Lyons MB, Rockwood CA. Hypoplasia of the glenoid. A review of sixteen patients. J Bone Joint Surg Am. 1993; 75(8):1175-1184.

[11] Resnick D, Walter RD, Crudale AS. Bilateral dysplasia of the scapular neck. AJR Am J Roentgenol. 1982; 139(2):387389.

[12] Cory DA, Fritsch SA, Cohen MD, Mail JT, Holden RW, et al. Aneurysmal bone cysts: imaging findings and embolotheraphy. AJR Am J Roentgenol. 1989; 153(2):369373.

[13] Kakarla S. Imaging of blow out metastases of bone-Pictorial essay. J Med Sci Res. 2016; 4(4):181-189.

[14] Robbin MR, Murphey MD, Temple HS, Kransdorf MJ, Choi JJ. Imaging of Musculoskeletal Fibromatosis. Radiographics. 2001; 21(33):585-599.

[15] Kakarla S. Musculoskeletal fluorosis- imaging spectrum, J Med Sci Res. 2013; 1(3):117-123.

[16] Kakarla S. Imaging in fibrous dysplasia. J Med Sci Res 2013; 1(2):74-81.

[17] Kakarla S. Paget disease of skeleton - Pictorial essay. J Med Sci Res. 2014; 2(1):34-40.

[18] IsaacJ, Shyamkumar NK, Karnik SV. Intraosseus schwannoma. J Postgrad Med. 2004; 50:127-128. 